\title{
OPTICAL ROTATORY DISPERSION AND ABSOLUTE CONFIGURATION-V.
}

\section{THE ABSOLUTE CONFIGURATION OF NATURAL PLASMALOGEN ${ }^{1}$}

\author{
J. Cymerman Crajg, D. P. G. hamon, K. K. Purushothaman and S. K. Roy \\ Department of Pharmaceutical Chemistry. \\ University of California, San Francisco, California 94122 \\ W. E. M. LANDS \\ Department of Biological Chemistry, University of Michigan, Ann Arbor, Michigan
}

(Received 16 December 1964; in revised form 23 July 1965)

\begin{abstract}
The 1-alkenylglycerol (III) and 1-alkylglycerol (II) obtained from natural plasmalogen (I) have been shown by optical rotatory dispersion measurements to have the same absolute configuration as natural chimyl and batyl alcohol. All compounds are L-l-glycerol ethers.
\end{abstract}

Plasmalogens are a group of aldehydogenic substances widely distributed in nature in both the animal and plant kingdom. ${ }^{2}$ Their chemical structure is that of a cis-1(alk-1'-enyl) ether of a 2-acyl-3-glycerylphosphate (I) where $\mathrm{R}^{\prime \prime}$ may be derived from choline, ethanolamine or serine. Hydrogenation and acid hydrolysis ${ }^{3}$ yielded chimyl and batyl alcohols (IIa and IIb), while enzymic hydrolysis followed by alkaline saponification ${ }^{3}$ afforded a cis-1-alkenylglycerol (III), the fatty aldehydes from which were shown to be almost completely saturated and to consist mainly of the palmitic and stearic derivatives.

The absolute configuration of the plasmalogens is of considerable biological and chemical interest in view of their possible biogenetic relationship ${ }^{4 a}$ to the corresponding diacylphosphatides which could be reduced to the plasmalogens, possibly via a hemiacetal intermediate. ${ }^{4 b}$ However, some disagreement exists on the optical properties of the 1-alkenyl-3-glycerylphosphates obtained by alkaline hydrolysis; thus specific rotations of $[\alpha]_{D}-8 \cdot 6^{\circ 5 a}$ and $+6 \cdot 25^{\circ s b}$ are recorded for 1-alkenyl-3-glycerylphosphorylethanolamine, and $-7 \cdot 85^{\circ 5 c}$ and "less than $2^{\circ{ }^{\circ} 2 b}$ for 1-alkenyl-3-glycerylphosphorylcholine. No optical rotations have been reported either for the 1-alkenyl(III) or for the 1-alkyl-glycerols (II) obtained by hydrolysis and hydrogenation of plasmalogens.

1 Supported (in part) by research grant HE-5881 from the National Institutes of Health, U.S. Public Health Service.

' For reviews: "E. Klenk and H. Debuch, in Progress in the Chemistry of Fats and other Lipids Vol. 6 Pergamon Press, Oxford (1963); ' M. M. Rapport and W. T. Norton, Ann. Rev. of Biochem. 31, 103 (1962).

2e H. Debuch, Z. Physiol. Chem. 317, 182 (1959); ' E. L. GotTfried and M. M. RAPPort, J. Biol. Chem. 237, 329 (1962); ' G. M. Gray, Biochem. J. 70, 425 (1958); ‘ S. J. Thannhauser, N. F. Boncoddo, and G. Schmidt, J. Biol. Chem. 188, 427 (1951); • H. R. Warner and W. E. M. Lands, J. Amer. Chem. Soc. 85, 60 (1963).

2s H. Goldfine and N. Baumann, Proc. Intern. Congr. Biochem. 6th; p. 574. New York (1964);

- J. C. Craig and E. C. Horning, J. Org. Chem. 26, 2098 (1960).

* M. M. Rapport, B. Lerner, N. Alonzo, and R. E. Franzl, J. Biol. Chem. 225, 859 (1957); ' S. J. Thannhauser, N. F. Boncoddo, and G. Schmidt, J. Biol. Chem. 188, 417 (1951); ' W. Bergmann and R. A. Landowne, J. Org. Chem. 23, 1241 (1958). 
Improved instrumentation has now made possible the direct determination of the absolute configuration of alcohols ${ }^{6}$ from their optical rotatory dispersion curve, and we wish to report the application of this method to the plasmalogens.

The 1-alkenyl-2-acylglycerol (from pig heart plasmalogen ${ }^{3 e}$ ) was hydrolysed to the 1-alkenylglycerol (III) ${ }^{30}$ and purified by chromatography on silicic acid. The IR and NMR spectra of this material agreed in all respects with those reported. ${ }^{36}$ Acetylation at room temperature gave the 1-alkenylglycerol 2,3-diacetate, and the NMR spectra of the acetylated and un-acetylated products possessed the same pattern for the olefinic protons of the vinyl ether linkage.

The optical rotatory dispersion curve of the alkenylglycerol diacetate (Fig. 1) could only be obtained down to $232 \mathrm{~m} \mu$, due to rising UV absorption by the vinyl
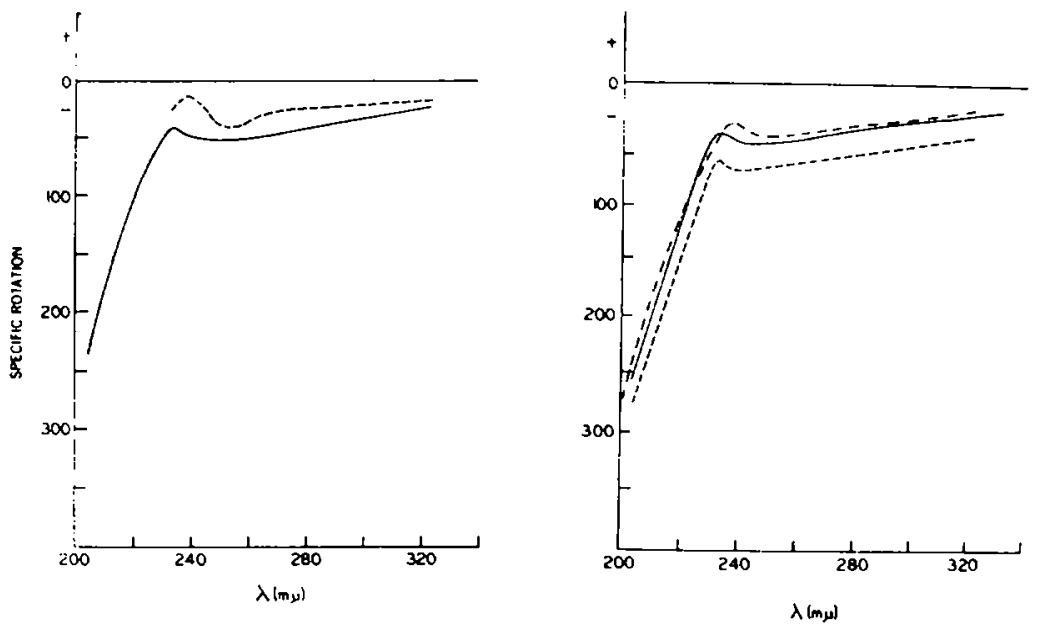

Fia. 1. Rotatory Dispersion Curves. (left) Alkenylglycerol diacetate (- - - ) and reduced alkenylglycerol diacetate $(-)$. (right) $(-)$-Chimyl diacetate $(-)$, $(-)$-batyl diacetate $(---)$, and (-)-batyl distearate $(-\cdot-)$.

ether chromophore. On hydrogenation it absorbed the theoretical amount of hydrogen and the alkylglycerol diacetate obtained had IR and NMR spectra identical with those of chimyl and batyl diacetates. Gas-liquid chromatography of the reduced alkenylglycerol diacetate gave two major peaks identified as chimyl diacetate $(70 \%$ by wt) and batyl diacetate ( $15 \%$ by wt) respectively, together with smaller amounts of homologues containing between 14 and 19 carbon atoms in the glycerol ether sidechain. Its rotatory dispersion curve (Fig. 1) is substantially identical with that of (-)-chimyl diacetate, and closely resembles those of (-)-batyl distearate and (-)-batyl diacetate (Fig. 1). The curves of the saturated diesters show a small positive Cotton effect superimposed upon a negative background, and resemble the R.D. curve of L-(-)-2octyl acetate. ${ }^{6}$ Thus both (-)-chimyl diacetate and L-(-)-2-octyl acetate show UV absorption at $216-218 \mathrm{~m} \mu$ and both give positive circular dichroism maxima at this wavelength.

Natural S-(+)-chimyl and batyl alcohol, and their 2,3-isopropylidene derivatives (IVa and b), exhibit plain negative curves like those of alcohols (e.g. L-(-)-2-octanol ${ }^{6}$ ) possessing the L-configuration with reference to $\mathrm{L}-(+)$-lactic acid. It should be noted

- J. C. Craig and S. K. Roy, Tetrahedron 21, 1847 (1965). 
that acylation of natural S-(+)-chimyl or batyl alcohol proceeds with retention of configuration (Fig. 1), as does the room temperature hydrolysis of the diacetate (Experimental), but that the operation of the sequence rule ${ }^{7}$ results in the reversal of the symbolism to give R-(-)-diesters. It is clear, however, that all compounds in Fig. 1 are L-1-glycerol ethers, in agreement with the reported ${ }^{8}$ conversion of $L-(-)-2,3-$ isopropylideneglycerol (obtained from L-(-)-glyceraldehyde), via L-(-)-1-O-bexadecyl-2,3-isopropylideneglycerol (IVa), into L-( + )-chimyl alcohol (IIa).

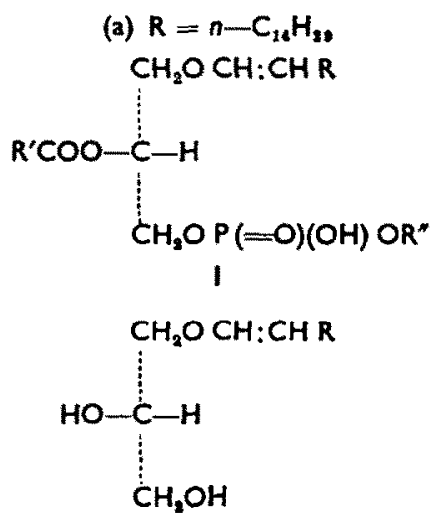

III

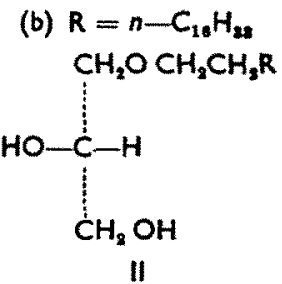<smiles>[R]COC[C@H]1COC(C)(C)O1</smiles>

IV<smiles>CC(O)COCCOCCN</smiles>

The results shown in Fig. 1 confirm that natural plasmalogen has the same configuration as natural chimyl and batyl alcohol. This conclusion supports the findings ${ }^{8}$ that synthetic $L-(-)-3-g l y c e r y l p h o s p h o r y l e t h a n o l a m i n e ~(V)$ of $[\alpha]_{D}-2 \cdot 9^{\circ}$ agreed well with the specific rotation $\left(-3 \cdot 26^{\circ}\right)$ found for the natural $(-)$-3-glycerylphosphorylethanolamine obtained ${ }^{10}$ from plasmalogen.

\section{EXPERIMENTAL}

Rotatory dispersion curves were determined with a Bendix model $460-\mathrm{C}$ spectropolarimeter using $1 \mathrm{~mm}$ or $1 \mathrm{~cm}$ cells $(c, 0.7$ to 2.6 in $95 \% \mathrm{EtOH})$ at $25^{\circ}$. Rotations are given below only for (1) the highest and lowest wavelengths measured, (2) peaks and troughs. Results were reproducible to within $5 \%$.

Natural alkenylglycerol diacetate. R.D. $\left(c, 1 \cdot 10\right.$ in EtOH) $[\alpha]_{322}-17 \cdot 6^{\circ},[\alpha]_{250}-40 \cdot 6^{\circ}$ (trough), $[\alpha]_{23 a}-15 \cdot 5^{\circ}$ (peak), $[\alpha]_{23 a}-27 \cdot 7^{\circ}$.

Reduced alkenylglycerol diacetate. R. D. $\left(c, 0.92\right.$ in EtOH) $[\alpha]_{s z 2}-23 \cdot 4^{\circ},[\alpha]_{1 s s}-41 \cdot 5^{\circ}$ (peak), $[\alpha]_{204}-238^{\circ}$. Gas-liquid chromatography was carried out using a column $(1.8 \mathrm{~m} \times 3 \mathrm{~mm})$ packed with $3 \%$ S.E. 30 siliconie on Gas-chrom $A$ at $225^{\circ}$ using argon ( 20 p.s.i.) as the carrier gas. The two

7a R. S. Cahn, C. K. Ingold and V. Prelog, Experientia 12, 81 (1956); ' R. S. Cahn, J. Chem. Education 41, 116 (1964).

to J. C. Sowden and H. O. L. Fischer, J. Amer. Chem. Soc. 63, 3244 (1941); ' E. Baer and H. O. L. Fischer, J. Biol. Chem. 140, 397 (1941).

- E. Baer and H. C. Stancer, J. Amer. Chem. Soc. 75, 4510 (1953).

${ }^{10} \mathrm{~S}$. J. Thannhauser, N. F. Boncoddo and G. Schmidt, J. Biol. Chem. 188, 423 (1951). 
main peaks were identified as chimyl diacetate ( $70 \%$ by wt. of total eluted material), retention time $4.96 \mathrm{~min}$, and batyl diacetate ( $15 \%$ by wt), retention time $8.02 \mathrm{~min}$.

Batyl Diacetate. $[\alpha]_{\mathrm{D}}-7.6^{\circ}\left(c, 9.2\right.$ in $\left.\mathrm{CHCl}_{\mathrm{s}}\right)$ R.D. $(c, 0.975$ in EtOH $)[\alpha]_{322}-43.7^{\circ},[\alpha]_{\mathbf{3 s}}$ $-65 \cdot 7^{\circ}$ (peak), $[x]_{204}-270^{\circ}$.

Chimyl diacetate. $[\alpha]-7.7^{\circ}\left(c, 5.7\right.$ in $\left.\mathrm{CHCl}_{3}\right)$. R.D. $\left(c, 0.724\right.$ in EtOH) $[\alpha]_{3 s 3}-24.8^{\circ},[\alpha]_{938}$ $-42 \cdot 1^{\circ}$ (peak), $[\alpha]_{204}-254^{\circ}$.

Batyl distearate. $[\alpha]_{\mathrm{D}}-4 \cdot 7^{\circ}\left(c, 6.25\right.$ in $\left.\mathrm{CHCl}_{2}\right)$. R.D. $(c, 2.625$ in hexane $)[\alpha]_{323}-24 \cdot 1^{\circ},[\alpha]_{283}$ $-38.0^{\circ}$ (peak), $[\alpha]_{200}-276^{\circ}$.

Chimyl alcohol. $[\alpha]_{\mathrm{D}} 3 \cdot 2^{\circ}\left(c, 2.57\right.$ in $\left.\mathrm{CHCl}_{\mathrm{s}}\right)$. R.D. $\left(c, 1.580\right.$ in EtOH $[\alpha]_{88 s}-18 \cdot 6^{\circ},[\alpha]_{204}-89 \cdot 0^{\circ}$. Batyl alcohol. $[x]_{\mathrm{D}} 4.7^{\circ}\left(c, 1.56\right.$ in $\left.\mathrm{CHCl}_{\mathrm{s}}\right)$. R.D. $(c, 1.005$ in $\mathrm{EtOH})[\alpha]_{3 s s}-7.75^{\circ},[x]_{204}-68 \cdot 7^{\circ}$. L-(-)-1-0-Hexadecyl-2,3-isopropylideneglycerol. $[\alpha]_{\mathrm{D}}-8 \cdot 3^{\circ}(c, 10 \cdot 7$ in EtOH). R.D. (c, 3.175 in EtOH) $[\alpha]_{3 s s}-35 \cdot 8^{\circ},[x]_{204}-228^{\circ}$.

L-(-)-1-0-Octadecyl-2,3-isopropylideneglycerol. $[\alpha]_{\mathrm{D}}-7 \cdot 5^{\circ}(c, 10 \cdot 1$ in EtOH). R.D. (c, 3.245 in EtOH) $[\alpha]_{s s s}-35 \cdot 0^{\circ},[\alpha]_{204}-228 \cdot 5^{\circ}$.

Hydrolysis of chimyl diacetate. Chimyl diacetate of $[\alpha]_{\mathbb{D}}-7.7^{\circ}$ (made from chimyl alcohol, $[\alpha]_{\mathrm{D}} 3 \cdot 2^{\circ}$ ) was allowed to stand overnight at room temp with $0 \cdot 1 \mathrm{~N} 90 \%$ alcoholic $\mathrm{NaOH}$ solution. Addition of NaClaq. and extraction with ether gave back chimyl alcohol whose m.p., mixed m.p., and specific rotation were identical with that of the chimyl alcohol used as the starting material. 